\title{
Low mitochondrial variability in birds may indicate Hill-Robertson effects on the W chromosome
}

\author{
S Berlin ${ }^{1,2}$, D Tomaras ${ }^{2}$ and B Charlesworth ${ }^{2}$ \\ ${ }^{1}$ Department of Genetics and Genomics, Roslin Institute (Edinburgh), Midlothian, UK and ${ }^{2}$ Institute of Evolutionary Biology, School of \\ Biological Sciences, University of Edinburgh, Edinburgh, UK
}

\begin{abstract}
Interference among loci subject to selection (the HillRobertson effect) may considerably reduce levels of adaptation and variability in genomic regions that lack recombination. $\mathrm{Y}$ - or $\mathrm{W}$ chromosomes are particularly vulnerable to such effects, since they represent large, non-recombining blocks of genetic material. In birds, the W chromosome and mitochondrial genomes are both maternally transmitted, and hence fail to recombine with each other, whereas in mammals the $Y$ chromosome is paternally transmitted. We
\end{abstract}

show here that mitochondrial DNA sequence diversity is reduced in non-ratite birds compared with mammals. After considering possible confounding factors, such as differences in generation times, mutation rates and demography, we conclude that Hill-Robertson effects associated with the $\mathrm{W}$ chromosome provide the most likely explanation for this difference.

Heredity (2007) 99, 389-396; doi:10.1038/sj.hdy.6801014; published online 6 June 2007

Keywords: W chromosomes; mitochondria; Hill-Robertson effects; birds; mammals

\section{Introduction}

Population genetics theory predicts that genomic regions with low or reduced recombination will exhibit reduced levels of adaptation and variability, caused by the action of selection at closely linked sites (the Hill-Robertson effect) (Hill and Robertson, 1966; Gordo and Charlesworth, 2001). This effect arises from the fact that any locus linked to another that is under directional selection experiences a reduction in effective population size $\left(N_{\mathrm{e}}\right)$. Since the efficacy of selection on a mutation is a function of the product of $N_{\mathrm{e}}$ and the effect of the mutation on fitness (Kimura, 1983), such linkage affects the probability of fixation of new mutations; favourable mutations are less likely to reach fixation and the opposite is true for deleterious mutations. Hill-Robertson effects can be caused by the spread to fixation of advantageous mutations (hitchhiking: (Maynard-Smith and Haigh, 1974; Gillespie, 2000)), the elimination of recurrent deleterious mutations (background selection: (Charlesworth et al., 1993)), the stochastic accumulation of deleterious mutations (Muller's ratchet: (Muller, 1964; Kim and Stephan, 2000)) and mutual interference between weakly selected mutations that spend long periods at intermediate frequencies (weak selection Hill-Robertson interference: (McVean and Charlesworth, 2000; Comeron and Kreitman, 2002)).

Recombination reduces the extent of this interference, increasing $N_{\mathrm{e}}$ and hence the efficacy of selection. We therefore expect lower rates of adaptive evolution, and

Correspondence: Dr S Berlin, Department of Evolutionary Biology, EBC, Uppsala University, Norbyvagen 18 D, Uppsala 75236, Sweden.

E-mail: sofia.berlin@ebc.uu.se

Received 20 November 2006; revised 19 April 2007; accepted 27

April 2007; published online 6 June 2007 higher rates of fixation of deleterious mutations, in genomic regions with low levels of genetic recombination, compared with more highly recombining regions. In addition, the level of variability at neutral or nearly neutral sites will also be reduced in low recombination genomes, since such variability is proportional to the product of $N_{\mathrm{e}}$ and the mutation rate (Kimura, 1983).

This prediction is consistent with a number of observations on molecular evolution and variation, with evidence for reduced levels of variability and adaptation in genomic regions with low levels of recombination (Gordo and Charlesworth, 2001; Presgraves, 2005). In particular, the degeneration of Y- and W chromosomes (in species with male and female heterogamety, respectively) is thought to result from this effect, since these have evolved as permanently heterozygous, non-recombining units (Charlesworth and Charlesworth, 2000). Support for this hypothesis is provided by data showing accelerated rates of protein sequence evolution and reduced levels of silent site DNA sequence diversity in systems such as the neo-Y chromosome of Drosophila miranda (Bachtrog, 2004), the evolving Y chromosome of Silene latifolia (Nicolas et al., 2005), and the $W$ chromosome of the chicken (Berlin and Ellegren, 2004, 2006).

Here we ask whether this observation holds generally in birds by using the fact that the mitochondrial (mt) genome of birds appears to be strictly maternally transmitted, and is thus completely associated with the W chromosome (Berlin and Ellegren, 2001; Berlin et al., 2004). If Hill-Robertson effects reduce W-chromosome diversity, then mtDNA diversity will also be reduced in birds. We test for such a reduction by comparing bird and mammalian mtDNA diversities, and evaluating possible confounding effects of differences in effective population size and mutation rates. 
It has recently been proposed that Hill-Robertson effects are so pervasive in animal mtDNA that its genetic diversity has little relation to population size (Bazin et al., 2006), in accordance with the theoretical proposal of Gillespie (2000). We show below that there are some patterns in DNA sequence variability in both bird and mtDNA that appear to be inconsistent with this idea; any Hill-Robertson effects that are acting seem to be too weak to entirely override the signature of demographic factors that influence effective population size.

\section{Materials and methods}

\section{Sequences and statistical analyses}

Mammalian and avian mitochondrial cytochrome $b$ sequences were collected from NCBI (http://www.ncbi. nlm.nih.gov/). This gene was chosen because it is the most widely sequenced coding region of the mitochondrial genome. One hundred and four mammalian species from 69 genera and 88 avian species from 61 genera were included in the study. We did not include avian species from the superorder Palaeognathae (ratites and tinnamous) because the Z- and W chromosomes of many of these species have only differentiated to a limited extent (Tsuda et al., 2007). All raw data along with references to each data set can be found as Supplementary Information Tables S1-S4. We generated two data sets each for birds and mammals; one 'total' data set and one 'population' data set, the latter containing sequences collected from a single location. Thus, each data point in the 'total' data set represents sequence information from one species (regardless of sampling location), whereas each data point in the 'population' data set is from one population (sequences sampled from one location). If there was more than one species per genus in the 'total' data set, the average value of the sequence diversity estimates per genus was used; if there was more than one population per species in the 'population' data set, a population average was used, and species averages were then used to get the genus average.

The nucleotide sequences were aligned by hand (no gaps). Intraspecific sequence variation was analysed in DnaSP 4.0 (Rozas et al., 2003). The pairwise nucleotide diversity, $\hat{\pi}$ (Nei, 1987), with Jukes Cantor correction (Jukes and Cantor, 1969), and Watterson's estimator $(\theta)$ (Watterson, 1975) were obtained for synonymous $\left(\hat{\pi}_{\mathrm{S}}, \theta_{\mathrm{S}}\right)$ and non-synonymous sites $\left(\hat{\pi}_{\mathrm{A}}, \theta_{\mathrm{A}}\right)$ separately for each species or population data set. These two estimates of the population parameter, $\theta=4 N \mu$, are expected to be the same for neutral sites at statistical equilibrium under mutation and genetic drift (Tajima, 1989). For alignments containing complex codons that differed by multiple substitutions, $\theta_{\mathrm{S}}$ and $\theta_{\mathrm{A}}$ were manually calculated, using the number of synonymous and replacement substitutions per synonymous and replacement sites respectively $(S)$, from the formula

$$
\hat{\theta}=S / a
$$

where $a$ is Watterson's correction factor, given by:

$$
a=\sum_{i=1}^{n-1} 1 / i
$$

and $n$ is the number of sequences in the sample (Watterson, 1975; Nei, 1987).
The variances of the values of $\hat{\pi}_{\mathrm{S}}$ and $\hat{\pi}_{\mathrm{A}}$ were obtained from the formula:

$$
\operatorname{var} \hat{\pi}=\frac{(n+1) \hat{\pi}_{\mathrm{G}}}{3(n-1) k}+\frac{2\left(n^{2}+n+3\right)}{9 n(n-1)} \hat{\pi}_{\mathrm{G}}^{2},
$$

where $k$ is the number of synonymous or non-synonymous sites, and $\hat{\pi}_{\mathrm{G}}$ is the overall unweighted genus mean (Nei, 1987, p 257). We used the genus mean rather than the sample value, since this should be closer to the expected value of $\hat{\pi}$, and hence avoids biases caused by low estimates of $\hat{\pi}$ for individual samples (Bartolomé et al., 2005).

The variances of the corresponding $\theta$ values were obtained from the following formula (Nei, 1987, p 255):

$$
\operatorname{var} \hat{\theta}=\left(\hat{\theta}_{\mathrm{G}} / k a\right)+\left(\hat{\theta}_{\mathrm{G}}^{2} b / a^{2}\right)
$$

where $\theta_{\mathrm{G}}$ is the overall unweighted genus mean $\theta$ for birds and mammals separately and $b$ is given by:

$$
b=\sum_{i=1}^{n-1} 1 / i^{2}
$$

Variance-weighted values of $\hat{\pi}_{\mathrm{S}}, \hat{\pi}_{\mathrm{A}}$ were then generated as the ratio of the means of $\hat{\pi} / \operatorname{var} \hat{\pi}$ to the mean of $1 / \operatorname{var} \hat{\pi}$ (and similarly for $\theta$ ), as described by Bartolomé et al. (2005). The standard errors of the mean diversity estimates across a set of genera were estimated as the standard deviation divided by the square root of the sample size (the number of genera).

The ratios $\hat{\pi}_{\mathrm{A}} / \hat{\pi}_{\mathrm{S}}$ and $\theta_{\mathrm{A}} / \theta_{\mathrm{S}}$ were calculated as the mean values of the diversity estimates for each genus. The variances of these ratios were estimated using the above formulae and the relation:

$$
\operatorname{var}\left(\hat{\pi}_{\mathrm{A}} / \hat{\pi}_{\mathrm{S}}\right) \approx\left(\operatorname{var} \hat{\pi}_{\mathrm{A}} / \hat{\pi}_{\mathrm{S}}^{2}\right)+\left(\hat{\pi}_{\mathrm{A}}^{2} \operatorname{var} \hat{\pi}_{\mathrm{S}} / \hat{\pi}_{\mathrm{S}}^{4}\right)
$$

All estimates were statistically compared by nonparametric Mann-Whitney $U$-tests, in Mini-Tab 14.20.

\section{Body masses}

Species were classified according to body mass, with one category containing species with body masses up to $100 \mathrm{~g}$ and another category including species with body masses from 100 to $6175 \mathrm{~g}$ (the largest bird). Large mammals with body masses over $6175 \mathrm{~g}$ were not used in any comparisons. Body mass measurements were taken from the literature (Dunning, 1993; Silva and Downing, 1995; Morrow and Fricke, 2004; Pitcher et al., 2005). When the literature reported a range of body masses, the means of these were used: $\hat{\pi}_{\mathrm{S}}, \hat{\pi}_{\mathrm{A}}, \hat{\pi}_{\mathrm{A}} / \hat{\pi}_{\mathrm{S}}, \theta_{\mathrm{S}}, \theta_{\mathrm{A}}$ and $\theta_{\mathrm{A}} / \theta_{\mathrm{S}}$ were then compared for mammals and birds within each body mass category. These estimates were also compared between passerines and bats. The mean values of $\hat{\pi}_{\mathrm{S}}, \hat{\pi}_{\mathrm{A}}$, $\hat{\pi}_{\mathrm{A}} / \hat{\pi}_{\mathrm{S}}, \theta_{\mathrm{S}}, \theta_{\mathrm{A}}$ and $\theta_{\mathrm{A}} / \theta_{\mathrm{S}}$ were also reported for each order.

\section{Population subdivision}

Average $F_{\mathrm{ST}}$ values per genera for birds and mammals, respectively, were estimated in DnaSP 4.0 (Rozas et al., 2003), using equation 3 in Hudson et al. (1992).

\section{Neutrality index}

The neutrality index (NI) (Rand and Kann, 1996) was estimated for sister taxa (more closely related to each other than to any other species in the data set) and then compared between mammals and birds. NI reflects the 
extent to which the levels of non-synonymous diversity depart from a strict neutral model and can be calculated as follows: $\left(\hat{\pi}_{\mathrm{A}} / \hat{\pi}_{\mathrm{S}}\right) /\left(K_{\mathrm{A}} / K_{\mathrm{S}}\right) . \mathrm{NI}=1$, indicates neutrality; $\mathrm{NI}>1$, indicate an excess of amino acid replacements within species; and $\mathrm{NI}<1$ indicates an excess of amino acid changes between species.

$\hat{\pi}_{\mathrm{A}}$ and $\hat{\pi}_{\mathrm{S}}$ were estimated in DnaSP, as described above. $K_{\mathrm{A}}$ and $K_{\mathrm{S}}$ are the non-synonymous and synonymous pairwise sequence divergences between related taxa, calculated using the maximum likelihood method implemented in codeml (runmode $=-2$, seqtype $=1$ ) in PAML 3.15 (Yang, 1997). PAML uses maximum likelihood to fit models of mutational changes among codons. Codeml implements the codon substitution model of Goldman and Yang (1994), which allows for arbitrary frequencies of all possible codons, a transition/ transversion mutational bias, and a parameter equivalent to $K_{\mathrm{A}} / K_{\mathrm{S}}$ that measures the relative rates of nonsynonymous and synonymous changes. The substitution rates are estimated by using the nucleotide frequencies at each codon position (the F3 $\times 4$ model of (Yang, 1997)) to obtain the expected codon frequencies at equilibrium under the substitution process.

In DnaSP, the total numbers of synonymous and nonsynonymous sites are computed by the method of Nei and Gojobori (1986), with a Jukes-Cantor correction for multiple changes. In contrast to the method of Goldman and Yang (1994), this procedure assumes that all nucleotide changes are equally frequent; two-thirds of changes at twofold degenerate positions are thus assumed to be synonymous. The numbers of nonsynonymous and synonymous sites in a sequence are estimated by weighting each site by the frequency with mutations are expected to result in the respective type of change, and the observed numbers of the two types of differences between two sequences are divided by the estimated numbers of sites to obtain the fractions of nonsynonymous and synonymous changes, respectively. Despite the fact that the two methods may give differences in the estimates of $K_{\mathrm{A}}$ and $K_{\mathrm{S}}$ themselves, the ratio $K_{\mathrm{A}} / K_{\mathrm{S}}$ is usually very similar, as we have established in a study of 22 genes in a comparison of Drosophila americana and D. ezoana (Maside and Charlesworth, unpublished). For either method, differences between species in levels of codon usage bias could result in differences in estimates of $K_{\mathrm{A}} / K_{\mathrm{S}}$ or $\hat{\pi}_{\mathrm{A}} / \hat{\pi}_{\mathrm{S}}$, but we found little evidence for a difference in codon usage between birds and mammals for cytochrome $b$ (data not shown).

\section{Results and discussion}

\section{Differences in mtDNA sequence diversity between mammals and birds}

Mean pairwise diversity per synonymous nucleotide site $\left(\hat{\pi}_{\mathrm{S}}\right)$ for mammalian genera ranged from 0 to 0.452 with a mean of $0.086 \pm 0.011$, and non-synonymous site diversity $\left(\hat{\pi}_{\mathrm{A}}\right)$ ranged from 0 to 0.0259 with a mean of $0.0036 \pm 0.0005$ (Table 1 ). $\hat{\pi}_{\mathrm{S}}$ in birds ranged between 0 and 0.1374 , with a mean of $0.0266 \pm 0.0045$, and $\hat{\pi}_{\mathrm{A}}$ ranged between 0 and 0.0158 with a mean of $0.0019 \pm 0.0003$ (Table 1 ). $\hat{\pi}_{\mathrm{S}}$ was significantly higher in mammals than in birds (Mann-Whitney $U$-test, $W=2840.5, \quad P<0.0001)$, as was $\hat{\pi}_{\mathrm{A}} \quad$ (Mann-Whitney

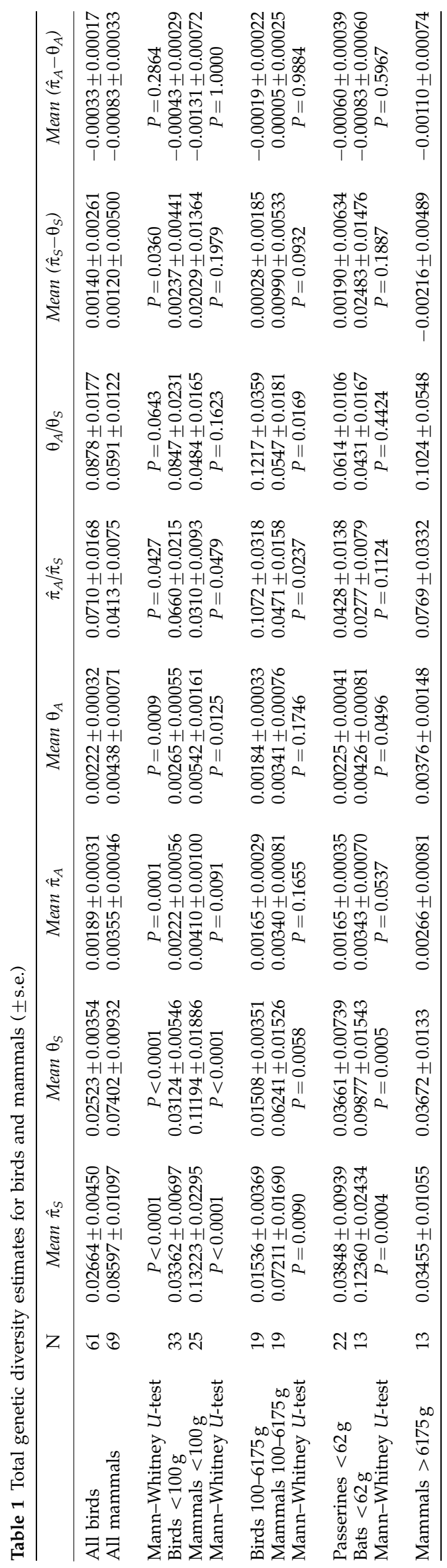




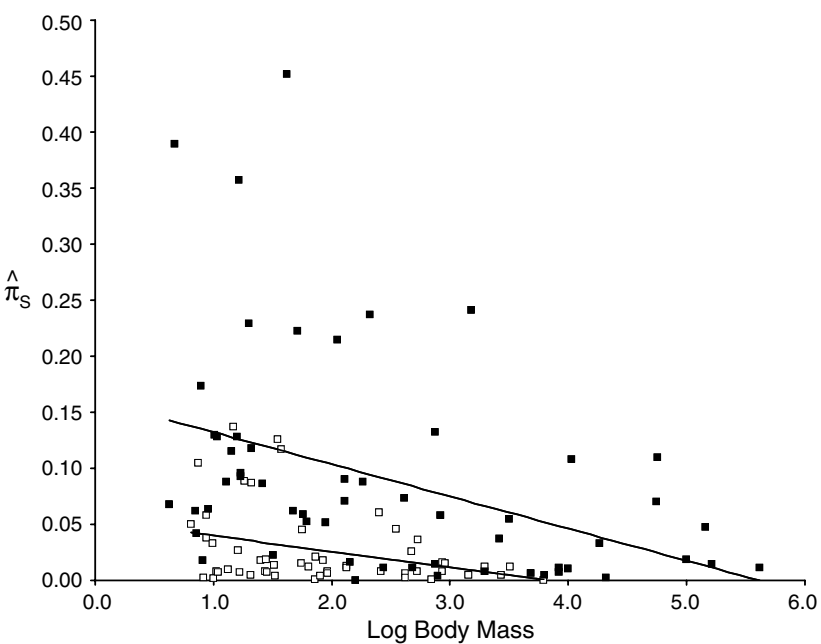

Figure 1 The mean values of synonymous diversity for genera of birds (open squares) and mammals (filled squares), plotted against the corresponding mean body masses. The regression lines, fitted as described in the text, are also shown.

$U$-test, $W=3169.5, P=0.0001$ ) (Table 1). Similar results are obtained with the $\theta$ estimates. Overall, therefore, there appears to be highly significantly lower mtDNA variability in birds than in mammals. Similar results are obtained when diversity is compared between bird and mammalian orders; both mean synonymous and nonsynonymous diversity are significantly lower for bird orders compared to mammalian orders $\left(\hat{\pi}_{\mathrm{S}}: W=117.0\right.$, $\left.P=0.017 ; \hat{\pi}_{\mathrm{A}}: \mathrm{W}: 123.0, P=0.044\right)$ (Table 2).

\section{Generation time effects on genetic diversity}

Difference in generation times are likely to affect differences in mtDNA variability between birds and mammals, since the neutral diversity for a given effective population size $\left(N_{\mathrm{e}}\right)$ is proportional to mutation rate per generation (Kimura, 1983), which is the product of generation time and mutation rate per year. To examine this effect, we divided the groups of species into two categories with respect to body size (less than $100 \mathrm{~g}$ and 100-6175 g) (Table 1). Body size correlates strongly with generation time (Finch, 1990; Charnov, 1993), and reliable data are more widely available for body size than generation time.

We found a significantly negative correlation between the genus averages synonymous diversity and mammalian body mass (Spearman's rank correlation: $\rho=-0.499$, $P<0.0001)$. The correlation for birds is in the same direction, but not as strong $(\rho=-0.295, P=0.034)$. We also fitted least squares regression lines to the bird and mammals separately, and used bootstrapping to obtain confidence intervals (CI), as described by Haddrill et al. (2005). Figure 1 shows that birds have a lower intercept than mammals $(0.030$ with $95 \%$ CI $0.021 / 0.040$ versus 0.095 with $95 \%$ CI $0.071 / 0.122)$, but a larger magnitude of regression coefficient $\left(-7.39 \times 10^{-6}\right.$ with $95 \%$ CI $-2.21 \times 10^{-5} /-4.01 \times 10^{-6}$ versus $-2.94 \times 10^{-7}$ with $95 \%$ CI $-1.06 \times 10^{-6}$ versus $-1.66 \times 10^{-7}$ ).

Overall, the results show that birds have significantly less synonymous site variability than mammals 
Table 3 Pairwise divergence and diversity estimates for birds

\begin{tabular}{|c|c|c|c|c|c|}
\hline Order & Species 1 & Species 2 & $\hat{\pi}_{A} / \hat{\pi}_{S}$ & $\mathrm{~K}_{A} / \mathrm{K}_{S}$ & $N I$ \\
\hline Apodiformes & Aerodramus maximus lowi & Collocalia esculenta cyanoptila & 0.1744 & 0.0153 & 11.4015 \\
\hline Coraciiformes & Atelornis pittoides & Todus todus & 0.1227 & 0.0173 & 7.0917 \\
\hline Galliformes & Lagopus mutus & Tetraogallus himalyensis & 0.2292 & 0.0078 & 29.3843 \\
\hline Gruiformes & Ardeotis arabs & Chlamydotis undulata macqueenii & 0.3477 & 0.0016 & 217.3405 \\
\hline Passeriformes & Acrocephalus bistrigiceps & Seicercus omeiensis & 0.0720 & 0.0159 & 4.5301 \\
\hline Passeriformes & Andropadus tephrolaemus & Hypsipetes amaurotis & 0.0117 & 0.0195 & 0.6009 \\
\hline Passeriformes & Basileuterus flavicauda & Vermivora ruficapilla & 0.0480 & 0.0170 & 2.8253 \\
\hline Passeriformes & Cacicus cela cela & Icterus galbula & 0.0493 & 0.0120 & 4.1100 \\
\hline Passeriformes & Calcarius lapponicus & Plectrophenax nivalis & 0.1031 & 0.0091 & 11.3295 \\
\hline Passeriformes & Corvus corax & Cyanopica cyanus pallescens & 0.1470 & 0.0117 & 12.5668 \\
\hline Passeriformes & Erithacus rubecula & Stiphrornis sanghensis & 0.0566 & 0.0166 & 3.4112 \\
\hline Passeriformes & Melospiza melodia & Spizella breweri & 0.0783 & 0.0241 & 3.2494 \\
\hline Passeriformes & Xiphorhynchus elegans & Empidonax traillii & 0.1388 & 0.0187 & 7.4240 \\
\hline Procellariiformes & Calonectis diomedea & Puffinus yelkouan & 0.1399 & 0.0137 & 10.2147 \\
\hline Psittaciformes & Cactua sulphurea & Pionopsitta barrabandi & 0.0641 & 0.0204 & 3.1418 \\
\hline Strigiformes & Aegolius funerus & Glaucidium brasilianum & 0.1477 & 0.0212 & 6.9658 \\
\hline Strigiformes & Bubo bubo & Strix aluco & 0.1412 & 0.0136 & 10.3790 \\
\hline
\end{tabular}

Mean

$0.1219 \pm 0.0194 \quad 0.0150 \pm 0.0013 \quad 20.3510 \pm 12.4144$

NI is the neutrality index: $\left(\hat{\pi}_{\mathrm{A}} / \hat{\pi}_{\mathrm{S}}\right) /\left(K_{\mathrm{A}} / K_{\mathrm{S}}\right)$, where $K_{\mathrm{A}}$ and $K_{\mathrm{S}}$ denote interspecies sequence divergences for non-synonymous and synonymous sites, respectively.

Table 4 Pairwise divergence and diversity estimates for mammals

\begin{tabular}{|c|c|c|c|c|c|}
\hline Order & Species 1 & Species 2 & $\hat{\pi}_{A} / \hat{\pi}_{S}$ & $\mathrm{~K}_{A} / \mathrm{K}_{S}$ & NI \\
\hline Carnivora & Martes americana & Mustela erminea & 0.0303 & 0.0138 & 2.1918 \\
\hline Carnivora & Panthera leo & Lynx canadensis & 0.1318 & 0.0283 & 4.6565 \\
\hline Cetartiodactyla & Bubalus bubalis & Pseudois nayaur & 0.0683 & 0.0268 & 2.5501 \\
\hline Cetartiodactyla & Camelus bactrianus & Lama guanicoe huanacus & 0.0709 & 0.0219 & 3.2354 \\
\hline Chiroptera & Lionycteris spurrelli & Lonchophylla robusta & 0.0206 & 0.0164 & 1.2580 \\
\hline Chiroptera & Mesophylla macconnelli & Vampyressa pusilla & 0.0275 & 0.0104 & 2.6399 \\
\hline Chiroptera & Myotis aurascens & Barbastella barbastellus & 0.0530 & 0.0198 & 2.6781 \\
\hline Insectivora & Blarina brevicauda & Sorex caecutiens & 0.0316 & 0.0119 & 2.6581 \\
\hline Primates & Alouatta caraya & Saimiri sciureus sciureus & 0.1837 & 0.0458 & 4.0115 \\
\hline Primates & Mandrillus sphinx & Macaca nemestrina nemestrina & 0.0605 & 0.0328 & 1.8450 \\
\hline Primates & Microcebus griseorufus & Lemur catta & 0.0393 & 0.0345 & 1.1396 \\
\hline Rodentia & Apodemus draco & Rhabdomys pumilio & 0.0507 & 0.0136 & 3.7243 \\
\hline Rodentia & Calomys fecundus & Scapteromys tumidus & 0.1861 & 0.0159 & 11.7061 \\
\hline Rodentia & Cavia tschudii & Dasy proctaleporina & 0.0714 & 0.0341 & 2.0932 \\
\hline Rodentia & Cratogeomys fumosus fumosus & Geomyspersonatus megapotamus & 0.0537 & 0.0154 & 3.4888 \\
\hline Rodentia & Glaucomyssabrinus griseifrons & Pteromys volans & 0.0476 & 0.0151 & 3.1501 \\
\hline Rodentia & Microtus oeconomus & Clethrionomys gapperi & 0.0216 & 0.0090 & 2.4017 \\
\hline Mean & & & $0.0676 \pm 0.0125$ & $0.0215 \pm 0.0025$ & $3.2605 \pm 0.5735$ \\
\hline
\end{tabular}

with comparable body sizes. A similar pattern is observed for non-synonymous variability, although the difference is not as pronounced. The most conservative comparison is probably that between small bats and small passerines, which are the species groups with the most similar generation times. Synonymous diversity is significantly lower in the passerines compared to the bats $(W=292.0, P=0.0004)$, and non-synonymous diversity is also lower ( $W=339.0, P=0.0537$ ) (Table 1 ). The relation between body size and diversity presumably reflect smaller $N_{\mathrm{e}}$ values for species with larger body size; this casts considerable doubt on the recent proposal that mtDNA is not affected by population size due to the prevalence of Hill-Robertson effects in the non-recombining mtDNA genome (Bazin et al., 2006).
Mutation rate differences between mammals and birds It is possible that mutation rates in mammalian mtDNA are intrinsically higher than in birds. Since there are no direct estimates of these mutation rates in birds, we have to rely on comparisons of estimates of synonymous or non-coding sequence divergence per year from mammals and birds, assuming these to be neutral (Kimura, 1983). It is currently unclear whether birds or mammals have higher rates of mtDNA sequence divergence (Garcia-Moreno, 2004; Ho et al., 2005; Lynch et al., 2006). A $2 \%$ rate of sequence divergence per million years is commonly used for mitochondrial coding genes for both mammals and birds (Brown et al., 1979), but there are many factors that could violate this general rule. Overall, there seems to be no evidence for differences that are large enough to overcome the 
differences in generation time between otherwise similar birds and mammals, given the fact that birds tend to have much longer generation times than mammals of comparable sizes (Finch, 1990). Other things being equal, this would tend to cause higher levels of variability in birds compared with mammals.

There is also indirect evidence that mutation rate differences cannot explain the difference in synonymous site diversity between birds and mammals that we have described. This comes from the results on non-synonymous variability (Tables 1 and 3 ). These estimates are much more similar for birds and mammals than the corresponding synonymous site variability estimates. In addition, $\theta_{\mathrm{A}}$ is nearly always greater than $\hat{\pi}_{\mathrm{A}}$, consistent with the action of purifying selection against deleterious amino acid site mutations that has been extensively documented before (Table 1) (Nachman, 1998; Fry, 1999; Weinreich and Rand, 2001). With such selection, a larger $N_{\mathrm{e}}$ has a much smaller effect on $\hat{\pi}_{\mathrm{A}}$ than $\hat{\pi}_{\mathrm{S}}$, since the former is more dependent on the ratio of mutation rate to selection coefficient than on $N_{\mathrm{e}}$, whereas neutral or nearly neutral diversity is proportional to $N_{\mathrm{e}}$ (Loewe et al., 2006).

If a difference in mutation rate were involved in the mammal-bird difference, both non-synonymous and synonymous diversity would be affected proportionately. In fact, overall $\hat{\pi}_{\mathrm{A}} / \hat{\pi}_{\mathrm{S}}$ is significantly higher in birds than mammals, and individual comparisons are always nominally higher (Table 1). This pattern is what is expected on the hypothesis of a reduction in the efficacy of selection in birds, due to Hill-Robertson effects that reduce $N_{\mathrm{e}}$ (Charlesworth and Charlesworth, 2000; Gordo and Charlesworth, 2001). Significantly higher NIs in birds (Table 3) (mean: 20.35 12.41) compared to mammals (mean: $3.26 \pm 0.57$ ) (Table 4) (W: 205.0, $P<0.0015)$ further support this conclusion. A high NI reflects less efficient selection against slightly deleterious mutations, which therefore become more abundant in the population but still have a low probability of fixation (Charlesworth, 1994; Rand and Kann, 1996). The average $K_{\mathrm{A}} / K_{\mathrm{S}}$ ratio is non-significantly higher in mammals than in birds ( $U$ : $103, P=0.15$ ). The theory of selection against slightly deleterious mutations predicts a much smaller effect of a reduction in $N_{\mathrm{e}}$ on $\hat{\pi}_{\mathrm{A}} / \hat{\pi}_{\mathrm{S}}$ than on $K_{\mathrm{A}} / K_{\mathrm{S}}$ (Charlesworth, 1994; Loewe et al., 2006), consistent with the lack of a significant difference in $K_{\mathrm{A}} / K_{\mathrm{S}}$ between birds and mammals.

\section{Demography and sequence variation}

The last possible confounding factor is the operation of demographic factors that cause lower $N_{\mathrm{e}}$ values in birds. Comparisons between nuclear gene diversities for birds and mammals should shed light on this possibility. Unfortunately, there are relatively few data on putatively neutral DNA sequence diversity in birds. The evidence suggests that birds have larger $N_{\mathrm{e}}$ values for nuclear genes than mammals. The ancestral $N_{\mathrm{e}}$ in a passerine species was recently estimated as several hundred thousand (Jennings and Edwards, 2005). The recent effective population size $\left(N_{\mathrm{e}}\right)$ in chicken is well above one million, using a mutation rate of $1.2 \times 10^{-9}$ (based on divergence data between chicken and turkey (Axelsson et al., 2004), a time of divergence of $38 \times 10^{6}$ (Dimcheff et al., 2002; van Tuinen and Dyke, 2004), and a generation

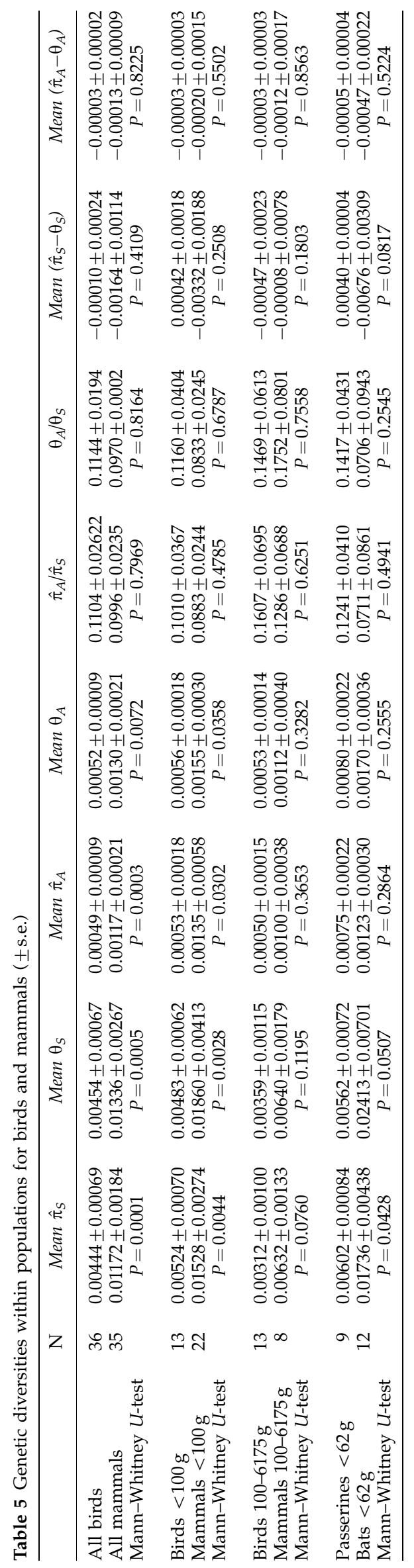


time of 1.5 years and average $\theta=7.7 \times 10^{-3}$ (Sundstrom et al., 2004)). In contrast, $N_{\mathrm{e}}$ in higher primates has been estimated as 10000-20000, and in rodents as 160000 (Eyre-Walker et al., 2002).

Population subdivision could, however, result in very different total $N_{\mathrm{e}}$ values for mtDNA and nuclear genes, as the $N_{\mathrm{e}}$ that controls diversity among genes sampled randomly from the species as a whole is inversely related to the rate of migration among local populations (Charlesworth et al., 2003). MtDNA is affected only by migration of females, and there is evidence that female mammals mostly disperse less than males, whereas the reverse is true for birds (Greenwood, 1980; Prugnolle and de Meeus, 2002). This difference in dispersal may contribute to the patterns we have reported. In order to examine this possibility, we estimated synonymous and non-synonymous diversities for sequences collected at the same locality. This provides estimates of genetic diversities within local populations, which are expected to be much less sensitive to migration rates than specieswide diversity levels (Charlesworth et al., 2003).

One hundred and thirty-four mammalian populations from 35 genera and 127 bird populations from 36 genera were analysed (Supplementary Material). Mean withinpopulation $\hat{\pi}_{\mathrm{S}}$ for mammalian genera ranged from 0.0017 to 0.0521 , with a mean of $0.0117 \pm 0.0018 ; \hat{\pi}_{\mathrm{A}}$ ranged from 0 to 0.0050 with a mean of $0.0012 \pm 0.0002$ (Table 5). Mean within-population $\hat{\pi}_{S}$ in bird genera ranged between 0 and 0.0209 , with a mean of $0.0044 \pm 0.0007$; and $\hat{\pi}_{\mathrm{A}}$ ranged between 0 and 0.0022 , with a mean of $0.0005 \pm 0.00009$. Both synonymous and non-synonymous diversities were significantly lower in birds than in mammals $\left(\hat{\pi}_{\mathrm{S}}: W=955.0, P=0.0001, \hat{\pi}_{\mathrm{A}}: W=1064.0\right.$, $P=0.0078$ ). We also grouped species by body mass; in most comparisons, $\hat{\pi}_{\mathrm{S}}$ was significantly lower in birds compared to mammals. $\hat{\pi}_{\mathrm{A}}$ was always lower in birds compared to mammals, although these differences were not statistically significant. We also compared passerines with bats, and again found lower $\hat{\pi}_{S}$ in the passerines compared to the bats.

We compared population structures in the mammals and the birds by estimating $F_{\mathrm{ST}}$ (Hudson et al., 1992; Charlesworth et al., 2003) for mammalian and bird species (averaging over species within genera). The average mammalian $F_{\mathrm{ST}}$ was $0.563 \pm 0.067$ and average bird $F_{\mathrm{ST}}$ was $0.384 \pm 0.068 \quad(P=0.077)$. These figures suggest that mammals may have a more pronounced population structure than birds with respect to mtDNA, although the difference is not statistically significant. A lower $N_{\mathrm{e}}$ for local populations, as expected if there are more intense Hill-Robertson effects on bird than mammalian mtDNA, would lead to increased $F_{\mathrm{ST}}$ (Charlesworth et al., 2003), so that this apparent difference is consistent with our hypothesis.

\section{Conclusions}

Overall, the finding that birds have lower mtDNA variability than mammals is most easily explained by Hill-Robertson effects acting on the $W$ chromosome. We recognize that this conclusion is only tentative, since this difference could be due to confounding factors for which we have not allowed. Further data on effective population sizes based on autosomal genes in birds and mammals, as well as direct measurements of
W-chromosome variability in a range of species, will provide additional data for testing this explanation further. Similar comparisons between other taxonomic groups with male and female heterogamety would also help to explore this question further.

\section{Acknowledgements}

We thank Martin Taylor for valuable discussion on the topic of population structure. This work was supported by a grant from the Royal Society to B Charlesworth and by a postdoctoral fellowship from the Swedish Research Council to S Berlin.

\section{References}

Axelsson E, Smith NGC, Sundstrom H, Berlin S, Ellegren H (2004). Male-biased mutation rate and divergence in autosomal, Z-linked and W-linked introns of chicken and turkey. Mol Biol Evol 21: 1538-1547.

Bachtrog D (2004). Evidence that positive selection drives Y-chromosome degeneration in Drosophila miranda. Nat Genet 36: 518-522.

Bartolomé C, Maside X, Yi S, Grant AL, Charlesworth B (2005). Patterns of selection on synonymous and non-synonymous variants in Drosophila miranda. Genetics 169: 1495-1507.

Bazin E, Glemin S, Galtier N (2006). Population size does not influence mitochondrial genetic diversity in animals. Science 312: $570-572$.

Berlin S, Ellegren H (2001). Evolutionary genetics - clonal inheritance of avian mitochondrial DNA. Nature 413: 37-38.

Berlin S, Ellegren H (2004). Chicken W: a genetically uniform chromosome in a highly variable genome. Proc Natl Acad Sci USA 101: 15967-15969.

Berlin S, Ellegren H (2006). Fast accumulation of nonsynonymous mutations on the female-specific $\mathrm{W}$ chromosome in birds. J Mol Evol 62: 66-72.

Berlin S, Smith NGC, Ellegren H (2004). Do avian mitochondria recombine? J Mol Evol 58: 163-167.

Brown WM, George M, Wilson AC (1979). Rapid evolution of animal mitochondrial DNA. Proc Natl Acad Sci USA 76: 1967-1971.

Charlesworth B (1994). The effect of background selection against deleterious mutations on weakly selected, linked variants. Genet Res 63: 213-227.

Charlesworth B, Charlesworth D (2000). The degeneration of Y chromosomes. Phil Trans $R$ Soc B 355: 1563-2572.

Charlesworth B, Charlesworth D, Barton NH (2003). The effects of genetic and geographic structure on neutral variation. Ann Rev Ecol Evol Syst 34: 99-125.

Charlesworth B, Morgan MT, Charlesworth D (1993). The effect of deleterious mutations on neutral molecular variation. Genetics 134: 1289-1303.

Charnov EL (1993). Life History Invariants. Some Explorations of Symmetry in Evolutionary Ecology. Oxford University Press: Oxford, UK.

Comeron JM, Kreitman M (2002). Population, evolutionary and genomic consequences of interference selection. Genetics 161: 389-410.

Dimcheff DE, Drovetski SV, Mindell DP (2002). Phylogeny of Tetraoninae and other galliform birds using mitochondrial $12 S$ and ND2 genes. Mol Phylogenet Evol 24: 203-215.

Dunning JB (1993). CRC Handbook of Avian Body Masses. CRC Press: Boca Raton, Florida.

Eyre-Walker A, Keightley PD, Smith NGC, Gaffney D (2002). Quantifying the slightly deleterious mutation model of molecular evolution. Mol Biol Evol 19: 2142-2149.

Finch CE (1990). Longevity, Senescence, and the Genome. University of Chicago Press: Chicago, IL. 
Fry AJ (1999). Mildly deleterious mutations in avian mitochondrial DNA: evidence from neutrality tests. Evolution $\mathbf{5 3}$ : 1617-1620.

Garcia-Moreno J (2004). Is there a universal molecular clock for birds? J Avian Biol 35: 465-468.

Gillespie JH (2000). Genetic drift in an infinite population. The pseudohitchhiking model. Genetics 155: 909-919.

Goldman N, Yang Z (1994). A codon-based model of nucleotide substitution for protein-coding DNA sequences. Mol Biol Evol 11: 725-736.

Gordo I, Charlesworth B (2001). Genetic linkage and molecular evolution. Curr Biol 11: R684-R686.

Greenwood PJ (1980). Mating systems, philopatry and dispersal in birds and mammals. Anim Behav 28: 1140-1162.

Haddrill PR, Thornton KR, Charlesworth B, Andolfatto P (2005). Multilocus patterns of nucleotide variability and the demographic and selection history of Drosophila melanogaster populations. Genome Res 15: 790-799.

Hill WG, Robertson A (1966). The effect of linkage on limits to artificial selection. Genet Res 8: 269-294.

Ho SY, Phillips MJ, Cooper A, Drummond AJ (2005). Time dependency of molecular rate estimates and systematic overestimation of recent divergence times. Mol Biol Evol 22 1561-1568.

Hudson RR, Boos DD, Kaplan NL (1992). A statistical test for detecting geographic subdivision. Mol Biol Evol 9: 138-151.

Jennings WB, Edwards SV (2005). Speciational history of Australian grass finches (Poephila) inferred from thirty gene trees. Evolution 59: 2033-2047.

Jukes TH, Cantor CR (1969). Evolution of protein molecules. In Munro HN (ed). Mammalian Protein Metabolism III. Academic Press: New York. pp 21-132.

Kim Y, Stephan W (2000). Joint effects of genetic hitchhiking and background selection on neutral variation. Genetics 155: 1415-1427.

Kimura M (1983). The Neutral Theory of Molecular Evolution Cambridge University Press: Cambridge.

Loewe L, Charlesworth B, Bartolomé C, Nöel V (2006). Estimating selection on nonsynonymous mutations. Genetics 172: 1079-1092.

Lynch M, Koskella B, Schaack S (2006). Mutation pressure and the evolution of organelle genomic architecture. Science 311 1727-1730.

Maynard-Smith J, Haigh J (1974). The hitch-hiking effect of a favourable gene. Genet Res 23: 23-35.

McVean GA, Charlesworth B (2000). The effects of HillRobertson interference between weakly selected mutations on patterns of molecular evolution and variation. Genetics 155: 929-944.

Morrow EH, Fricke C (2004). Sexual selection and the risk of extinction in mammals. Proceedings 271: 2395-2401.
Muller HJ (1964). The relation of recombination to mutational advance. Mutat Res 106: 2-9.

Nachman M (1998). Deleterious mutations in animal mitochondrial DNA. Genetica 102/103: 61-69.

Nei M (1987). Molecular Evolutionary Genetics. Columbia University Press: New York.

Nei M, Gojobori T (1986). Simple methods for estimating the numbers of synonymous and nonsynonymous nucleotide substitutions. Mol Biol Evol 3: 418-426.

Nicolas M, Marais G, Hykelova V, Janousek B, Laporte V, Vyskot B et al (2005). A gradual process of recombination restriction in the evolutionary history of the sex chromosomes in dioecious plants. PLoS Biology 3: 47-56.

Pitcher TE, Dunn PO, Whittingham LA (2005). Sperm competition and the evolution of testes size in birds. I Evol Biol 18: 557-567.

Presgraves DC (2005). Recombination enhances protein adaptation in Drosophila melanogaster. Curr Biol 15: 1651-1656.

Prugnolle F, de Meeus T (2002). Inferring sex-biased dispersal from population genetic markers: a review. Heredity 88: 161-165.

Rand DM, Kann LM (1996). Excess amino acid polymorphism in mitochondrial DNA: contrasts among genes from Drosophila, mice, and humans. Mol Biol Evol 13: 735-748.

Rozas J, Sanchez-DelBarrio JC, Messeguer X, Rozas R (2003). DnaSP, DNA polymorphism analyses by the coalescent and other methods. Bioinformatics 19: 2496-2497.

Silva MB, Downing JA (1995). CRC Handbook of Mammalian Body Masses. CRC Press: Boca Raton, Florida.

Sundstrom H, Webster MT, Ellegren H (2004). Reduced variation on the chicken $\mathrm{Z}$ chromosome. Genetics 167: 377-385.

Tajima F (1989). Statistical method for testing the neutral mutation hypothesis. Genetics 123: 585-595.

Tsuda Y, Nishida-Umehara C, Ishijima J, Yamada K, Matsuda Y (2007). Comparison of the $Z$ and $W$ sex chromosomal architectures in elegant crested tinamou (Eudromia elegans) and ostrich (Struthio camelus) and the process of sex chromosome differentiation in palaeognathous birds. Chromosoma 116: 159-173.

van Tuinen M, Dyke GJ (2004). Calibration of galliform molecular clocks using multiple fossils and genetic partitions. Mol Phyl Evol 30: 74-86.

Watterson GA (1975). On the number of segregating sites in genetical models without recombination. Theor Popul Biol 7: 256-276.

Weinreich DM, Rand DM (2001). Contrasting patterns of nonneutral evolution in proteins encoded in nuclear and mitochondrial genomes. Genetics 156: 385-399.

Yang Z (1997). PAML: a program package for phylogenetic analysis by maximum likelihood. Comput Appl Biosci 13: 555-556.

Supplementary Information accompanies the paper on Heredity website (http://www.nature.com/hdy) 